\title{
A Travel Route Recommendation Algorithm based on Interest Theme and Distance Matching
}

\section{Xi Cheng ( $\sim 20030032 @ s a s u . e d u . c n)$}

Sichuan University of Arts and Science

\section{Research}

Keywords: Travel route recommendation, interest theme, user interest, distance matching

Posted Date: May 17th, 2021

DOl: https://doi.org/10.21203/rs.3.rs-507597/v1

License: (c) (i) This work is licensed under a Creative Commons Attribution 4.0 International License. Read Full License

Version of Record: A version of this preprint was published at EURASIP Journal on Advances in Signal Processing on August 3rd, 2021. See the published version at https://doi.org/10.1186/s13634-02100759-x. 


\title{
A travel route recommendation algorithm based on interest theme and distance matching
}

\author{
Xi Cheng \\ Sichuan University of Arts and Science,Dazhou 635000, China.
}

20030032@sasu.edu.cn

\begin{abstract}
To solve the problem of low accuracy of traditional travel route recommendation algorithm, a travel route recommendation algorithm based on interest theme and distance matching is proposed in this paper. Firstly, the real historical travel footprints of users are obtained through analysis. Then, the user's preferences of interest theme and distance matching are proposed based on the user's stay in each scenic spot. Finally, the optimal travel route calculation method is designed under the given travel time limit, starting point and end point. Experiments on the real data set of the Flickr social network showed that the proposed algorithm has a higher accuracy rate and recall rate, compared with the traditional algorithm that only considers the interest theme and the algorithm which only considers the distance matching
\end{abstract}

Keywords: Travel route recommendation, interest theme, user interest, distance matching

\section{Introduction}

In recent years, research work in the field of recommended systems has developed rapidly. A variety of recommendation systems have also been widely used in a large number of fields such as e-commerce, social networking sites, e-tourism, Internet advertising, etc. And these recommendation systems show superior effects and prospects[1-3]. With the rise of more and more online travel websites (such as Expedia, Travelzoo, Tuniu, etc.), online data that can describe users' interest preferences is becoming more and more abundant. This makes the recommendation of tourism products become one of the hot spots in the research of recommendation system[4-5].

At present, many mature recommendation algorithms have been widely used for the recommendation of traditional commodities, such as collaborative filtering algorithm[6], content-based recommendation algorithm[7] and hybrid recommendation algorithm[8]. However, a large number of existing studies have shown that recommendation of tourism products is significantly different from traditional recommendation of films and commodities[9-11], and the difference is described as following.

First, users typically do not buy travel products frequently or in bulk, which results in the "user-product" correlation matrix being extremely sparse. Second, the description of tourism product information is diverse and complex, and small parameter changes will lead to completely different tourism products, such as changes in attractions and schedules, hotel and vehicle choices. However, such different tourism products with intrinsic relevance point to the common interest preferences of users. Third, users often do not pay attention to tourism products for a long time, that is, leave an access record on the electronic tourism website, and often after browsing targets and arrangements. Then they begin to browse the tourism

\footnotetext{
* Corresponding author.

E-mail address:20030032@sasu.edu.cn.
} 
products, which leads to a large number of cold-start users in the online travel data. Therefore, it is difficult to apply the traditional recommendation algorithm to tourism recommendation. In general, the above related recommendation techniques have solved the travel recommendation problem to some extent. However, such recommendation technology only applies to tourism data with relatively simple data structure, or relies on geographic information data, and it is difficult to comprehensively capture users' real-time interest preferences.

The data used in this paper is the real Web server log of tourism enterprises, which contains rich information related to tourism products and a large number of click records of user behaviors. The recommendation engine can be built to accurately capture users' interests based on their real-time click streams. Then, personalized travel products are recommended to users according to their interests. In order to improve the accuracy of the recommendation system, a travel route recommendation algorithm based on the topic of interest and distance matching is proposed. The core idea is to calculate the user's preference theme and acceptable distance based on the travel history data of each user, and add them into the recommendation model as weights to obtain a novel personalized travel route recommendation algorithm.

The rest of the paper is organized as follows. We review the related works in section one. The definition of necessary concepts and the proposed algorithm are presented in section two. Section three gives the experimental results. At last, section four draws a conclusion of the paper.

\section{The related work}

With the continuous development of social networks, users' social network information is increasing. How to effectively dig out valuable information from social network information plays an irreplaceable role in the development of social network [12]. In the social network, users can upload text information, location information and time information, and can also share this information with friends and people nearby. Nowadays, more and more scholars have realized the importance of social network information and have been devoting themselves to the research of social network information mining.

The idea of social network data mining is similar to that of GPS trajectory data mining. In GPS trajectory data mining, the main applications include association rules, abnormal behaviors, travel modes and GPS trajectory recommendation [13]. The data acquisition time has a strict limitation of equal time interval, which is reflected by SHAHED[14]. In social network trajectory data mining, applications mainly include location recommendation, route recommendation and behavioral preference recommendation [15]. The data acquisition time has dispersion and randomness, which are also the main differences between social network trajectory data and GPS trajectory data.

At present, there are many processing methods in social network data mining, mainly including traditional techniques such as clustering and classification in data mining. Among them, clustering method is used to find out the group pattern mining method in social networks, which has a good effect on recommending user routes and locations. The MapReduce [16] framework is widely used for large-scale data processing. At present, the method of combining clustering algorithm with MapReduce framework for big data analysis and processing has been gradually developed, such as DBSCAN clustering algorithm based on MapReduce, which has achieved good results.

The mining methods of group patterns mainly include swarm, flock, convoy, gathering, and platoon, etc. The literature [17] introduces in detail different mining methods of group patterns. Swarm is a group pattern mining technology with weak 
time-axis constraints, and it only needs to satisfy the condition that the number of times of different tracks appearing at the same point at the same time is greater than the set threshold. While flock and convoy are more time-constrained than swarm, this strong constraint also leads to a decline in accuracy. The literature [18] details the platoon model. The Platoon model combines the advantages of the above group models and adapts to different applications by allowing control of continuous time constraints.

Personalized recommendation methods mainly include content-based recommendation, collaborative filtering recommendation, association rule based recommendation, utility based recommendation, knowledge based recommendation and combined recommendation [19]. At the same time, there are many recommendation strategies, and different recommendation strategies produce different recommendation results. However, in the group pattern-based personalized travel route recommendation, the traditional group pattern mining leads to incomplete personalized recommendation due to the lack of semantic information.

\section{Methods}

\subsection{Basic definition}

Given a directed weighted graph $G=(E, V), V$ is the set of nodes, and $E$ is the set of edges. As shown in figure 1 , a node $p \in V$ represents a $P O I$. Each $P O I_{p}$ has category attributes $\mathrm{Cat}_{p}$ (such as church, museum, beach), longitude and latitude, and the value on node $p$ represents the score of $P O I_{p} . C$ represents a collection of categories of all $P O I S$. In the attribute $\left(c_{i}, D_{i}\right)$ of the node $p_{i}, c_{i}$ represents the category attribute of the $P O I$, and $D_{i}$ represents the distance of the POI. Each directed edge $\left(p_{i}, p_{j}\right)$ represents a feasible route between two $P O I s$, the number of edges is $|E|$, and the weight on the edge represents the travel time (in h) of the continuous access to the two $P O I \mathrm{~s}$.

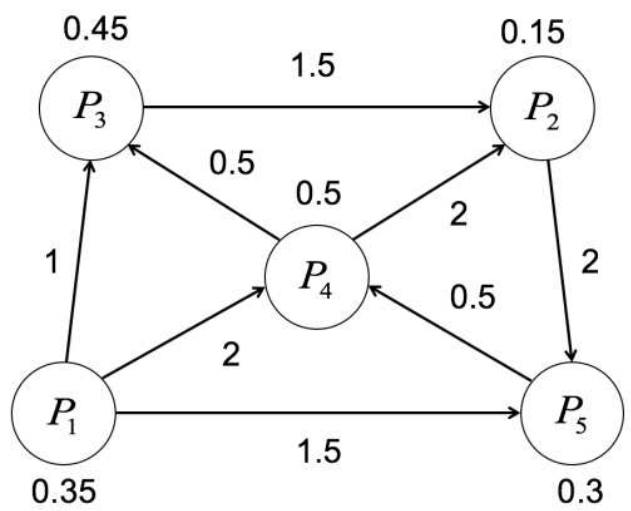

Figure 1 An example of a route recommendation 
A travel route is a sequence consisting of multiple travel POIs, denoted as $R=\left\{p_{1}, p_{2}, \ldots, p_{N}\right\}$. Where $p_{i}$ is the tourist location included in the route and $\mathrm{N}$ is the number of locations.

The travel time between two POIs. The travel time required by the user from $P O I_{P_{x}}$ to $P O I_{P_{y}}$ can be defined as follows,

$$
T^{\text {Travel }}\left(p_{x}, p_{y}\right)=\operatorname{Dist}\left(p_{x}, p_{y}\right) / \text { speed }
$$

Where $\operatorname{Dist}\left(p_{x}, p_{y}\right)$ represents the distance between $\mathrm{p}_{x}$ and $\mathrm{p}_{y}$, which is calculated by Haversine formula [20]. Suppose the user walks to play and takes a speed of $6 \mathrm{~km} / \mathrm{h}$.

The preference vector of a user $u$ is expressed as $\operatorname{Int} P(u)=\left\langle\operatorname{Int}\left(u, c_{1}\right), \operatorname{Int}\left(u, c_{2}\right), \ldots, \operatorname{Int}\left(u, c_{i}\right)\right\rangle$, Where $\operatorname{Int}\left(u, c_{i}\right)$ represents the degree of preference of the user $u$ for the $P O I$ category $c_{i}$.

Given a user $u$ and the $P O I$ collection he/she has been to, define his/her historical travel footsteps in chronological order $S_{u}=\left(\left(\mathrm{p}_{1}, t_{p_{1}}^{a}, t_{p_{1}}^{d}\right),\left(p_{2}, t_{p_{2}}^{a}, t_{p_{2}}^{d}\right) \ldots,\left(p_{n}, t_{p_{n}}^{a}, t_{p_{n}}^{d}\right)\right)$. Each triplet $\left(p_{x}, t_{p_{x}}^{a}, t_{p_{x}}^{d}\right)$ consists of a $P O I_{P_{x}}$ that the user has visited, a time $t_{p_{x}}^{a}$ that reaches $p_{x}$, and a time $t_{p_{x}}^{d}$ that leaves $p_{x}$, consisting of three elements. The first photo taken by the user in each $P O I$ is the time of the user's arrival and the last photo is the time of the user's departure. The user's access time in $p_{x}$ (that is, the user u's stay in $p_{x}$ ) can be valued by the difference between $t_{p_{x}}^{a}$ and $t_{p_{x}}^{d}$. Similarly, for the travel sequence $S_{u}, t_{p_{1}}^{a}$ and $t_{p_{n}}^{d}$ represent the start and end times of the journey respectively. For simplicity, this paper represents $S_{u}=\left(\left(p_{1}, t_{p_{1}}^{a}, t_{p_{1}}^{d}\right),\left(p_{2}, t_{p_{2}}^{a}, t_{p_{2}}^{d}\right) \ldots,\left(p_{n}, t_{p_{n}}^{a}, t_{p_{n}}^{d}\right)\right)$ as $S_{u}=\left(p_{1}, p_{2}, \ldots, p_{n}\right)$

The travel footprint $S_{u}=\left(p_{1}, p_{2}, \ldots, p_{n}\right)$ of the user $u$ is known, and if $t_{p_{x+1}}^{a}-t_{p_{x}}^{d}>\tau, S_{u}$ is divided into a number of individual travel sequences (that is, sub-sequences of $S_{u}$ ). In other words, if the time between consecutive accesses of two POIS is greater than the threshold $\tau$, the travel footprint is divided into a number of different tourist subsequences. The time threshold value $\tau$ is selected in this document as $8 \mathrm{~h}$. 
This paper gives a $P O I$ scoring method considering location distance and user preferences. The score for $P O I_{p_{i}}$ is

expressed as $\operatorname{score}\left(p_{i}\right)$

$$
\operatorname{score}\left(p_{i}\right)=\alpha \times \operatorname{Int}\left(u, c_{i}\right)+(1-\alpha) D\left(p_{i}\right)
$$

where $c_{i}$ is the category of $P O I_{p_{i}}, D\left(p_{i}\right)$ is the distance of $P O I_{p_{i}}$, and $\alpha$ is the user adjustment parameter, which is used to adjust the proportion of user interest preference and $P O I$ distance in the route.

\subsection{Tourist route recommendation framework}

As shown in Figure 2, the travel route recommendation in this paper is divided into the construction of the POI association graph and the learning of the user's interest preference, as well as the route recommendation. The construction of the $P O I$ association graph and the learning of the user's interest preference are performed offline, and the distance of the $P O I$ and the user's interest preference can be obtained by analyzing the photos taken by the user. The route recommendation is online, assuming that the user wants to go to the city with $m$ POIs, recorded as $P=\left\{p_{1}, p_{2}, \ldots, p_{m}\right\}$.

According to the $P O I$ set $P$, time budget $B$, starting point $P O I_{p_{1}}$ and ending point $P O I_{p_{n}}$, the route with the highest score is recommended to users by using the proposed algorithm which combines user interest preferences and POI distance based on the orientation problem.
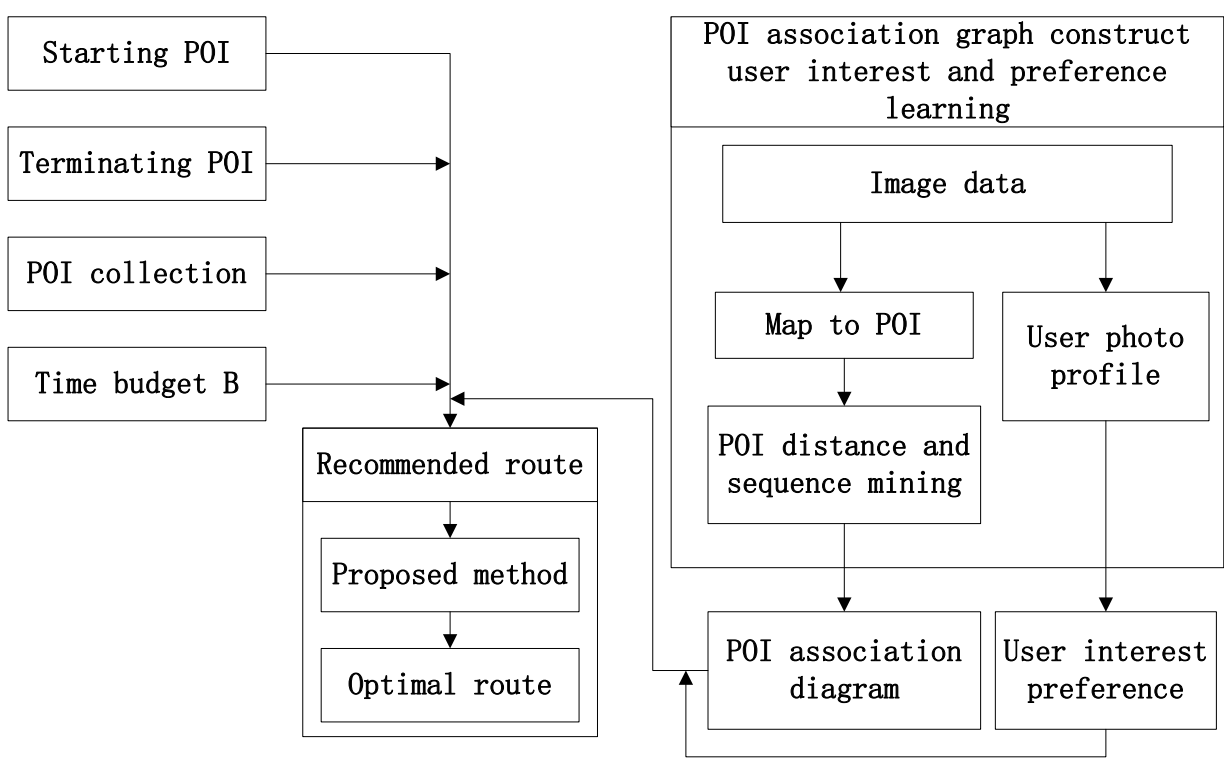
Figure 2 Route recommendation framework

\subsection{Construction of POI correlation graph}

The construction of the POI association diagram takes place offline. In this paper, POI in the tourism sequence of all users is used as the node in the graph, which represents the tourism place, and the continuous access of users in the tourism sequence generates the edges in the graph.

The structure of the photo data shared by the user is (PhotoID, UserID, Time, Longitude, Latitude, Category). From this structure, the photo data contains the exact space-time location information of the user. Based on the longitude and latitude of each photo, Haversine formula [20] is used to calculate the distance between each photo shared by the user and each POI in the city visited. If the distance is less than $200 \mathrm{~m}$, the photograph is considered to be taken at POI, so as to obtain the list of users POI $S_{u}=\left(p_{1}, p_{2}, \ldots, p_{n}\right)$.

A time based user interest preference is presented by using the user 's historical travel footprint. When a user goes to a POI to play, he/she will stay at the POI for a certain period of time. The access time (ie, the stay time) of each POI that each user has visited is calculated from the historical travel footprint of all users according to definition 4, so that the average time required for any user to access any one of the POIs can be calculated. In the travel route recommendation in this article, $\bar{V}(p)$ is used to indicate the average access time of his/her $P O I_{p}$ for any user. The average access time required for each $P O I_{p}$ is as follows

$$
\bar{V}(p)=\frac{1}{n} \sum_{u \in U} \sum_{p_{x} \in S_{u}}\left(t_{p_{x}}^{d}-t_{p_{x}}^{a}\right) \sigma\left(p_{x}=p\right) ; \forall p \in P
$$

Where $U$ represents all users, and $n$ represents the number of users accessing $p$ in $U . \sigma\left(p_{x}=p\right)=\left\{\begin{array}{c}1, p_{x}=p \\ 0, \text { other }\end{array}\right.$.

However, the average access time of the user at each POI does not truly reflect his/her degree of interest preference for this type of POI. Therefore, a time-based user interest preference is proposed in this paper. The preference degree of user $u$ to category attribute $c$ of POI is calculated from the following equation.

$$
\operatorname{Int}(u, c)=\sum_{p_{x} \in S_{u}} \frac{\left(t_{p_{x}}^{d}-t_{p_{x}}^{a}\right)}{\bar{V}\left(p_{x}\right)} \sigma\left(C a t_{p_{x}}=c\right) ; \forall c \in C
$$


Where Cat $t_{p}$ represents the category attribute of POIp, $\sigma\left(\right.$ Cat $\left._{p_{x}}=c\right)=\left\{\begin{array}{c}1, C_{p_{x}}=c \\ 0, \text { other }\end{array}\right.$. The above equation determines the interest of user $u$ in the category attribute $c$ for a particular POI. Relative to the average access time of all users in the same POI, it is calculated based on the time spent by users in each POI with category attribute $c$. In other words, a user may spend more time accessing the type of POI he or she is interested in, which in turn determines the user's level of interest in such POI.

\subsection{Proposed algorithm}

OP (Orienteering Problem) is directional Problem, which is described as follows. In a directed weighted graph G(V,E), V is the set of all points on the graph, and $\mathrm{E}$ is the set of all edges on the graph. Each point has its score (score, which can be expressed as gain), and each edge has its weight (weight, which is the walking time between two points). The start and end points are specified. Select partial points from G. Then plan a path through the selected points, the starting points and ending points. At the same time, under the premise of not exceeding a certain time budget, the total weight score of the path is maximized.

OP has been widely used in travel route recommendations. The route recommendation algorithm is proposed in consideration of POI distance and user interest based on the orientation problem. Based on the set $\mathrm{P}$, the time budget $\mathrm{B}$, the starting point $P O I_{p_{1}}$ and the end point $P O I_{p_{n}}$, the proposed algorithm recommends a route $R=\left\{p_{1}, p_{2}, \ldots, p_{n}\right\}$ that satisfies the time budget $\mathrm{B}$ and has the highest score. The time budget is calculated by function $\operatorname{Cost}\left(p_{x}, p_{y}\right)$, $\operatorname{Cost}\left(p_{x}, p_{y}\right)=T^{\text {Travel }}\left(p_{x}, p_{y}\right)+\bar{V}\left(p_{y}\right)$. Therefore, the travel route recommendation model in this paper can satisfy the integer programming problem with multiple constraints, which is expressed as follows

$$
\operatorname{Max} \sum_{i=2}^{N-1} \sum_{j=2}^{N} x_{i, j} \operatorname{score}\left(p_{i}\right)
$$

where $x_{i, j}=1$ indicates the route from $i$ to $j$, which goes through the edge $\left(p_{i}, p_{j}\right)$, otherwise $x_{i, j}=0$. The above equation satisfies the following constraints

$$
\begin{gathered}
\sum_{j=2}^{N} x_{1, j}=\sum_{i=1}^{N-1} x_{i, N}=1 \\
\sum_{j=2}^{N} x_{k, j}=\sum_{i=1}^{N-1} x_{i, k} \leq 1 ; \forall k=2,3, \ldots, N-1
\end{gathered}
$$




$$
\begin{gathered}
\sum_{i=1}^{N-1} \sum_{j=2}^{N} \operatorname{Cost}(i, j) x_{i, j} \leq B \\
2 \leq u_{i} \leq N \\
u_{i}-u_{j}+1 \leq(N-1)(1-x) ; \forall i, j=2,3, \ldots, N
\end{gathered}
$$

Equation (5) is an objective function that maximizes the POI distance and user interest preferences in the recommended route. Equations (6) to (10) are the constraints of equation (5). Equation (6) ensures that the user's travel starting point is $p_{1}$ and the end point is $p_{n}$. Equation (7) ensures that the user's travel route is coherent and that each POI in the route has only been visited once. Equation (8) guarantees that the time spent by the user on the entire trip is within budget $B$. Assuming that $u_{x}$ is the location of POIx in route $R$, Eq. (9) and Eq. (10) ensure that there are no sub-patrol routes in the integer programming problem proposed in this paper.

\section{Experimental}

\subsection{Experimental data and the data preprocess}

The Flickr dataset of the WAMDM (Web And Mobile Data Management) is used in this paper, which contains two aspects of content. One is 319,110 photos of New York City, including photo ID (Identification), photo shooting location (latitude and longitude), shooting time, photo tag information and user ID. The other is the address book of 12,991 Flickr users. In this experiment, the leap-one-out cross validation method[21], which is used in most recommendation system studies, is adopted to carry out experimental verification on the algorithm. It circulates one data in the whole data set as the test set, and other data as the training set, and calculates various evaluation indexes based on the prediction results of each cycle.In order to facilitate the experiment, the data is processed as follows. 1) Delete users who have taken fewer than 5 photos in each POI. 2) Delete users who have only visited one or two POIs. After data pre-processing, the final experimental data set consists of 5,5451 pictures taken by users with a geographical label 165, 830 pictures.

\subsection{Evaluation index}

The accuracy of recommendation is the most important index of evaluation algorithm. In this paper, precision and recall are used as criteria for measuring the pros and cons of the algorithm, which are expressed as follows. 


$$
\begin{gathered}
\text { precision }=\left|\begin{array}{ll}
P_{r} & P_{v}
\end{array}\right| /\left|P_{r}\right| \\
\text { recall }=\left|P_{r} \mathrm{I}_{v} P_{v}\right| /\left|P_{v}\right|
\end{gathered}
$$

Precision represents the probability that the user is interested in the recommended route, and recall represents the probability that a user's favorite POI is recommended. The higher the accuracy and recall, the better the recommended effect. $P_{r}$ represents the POI set in the recommended route, and $P_{v}$ represents the POI set visited by users in the real tourism sequence.

\section{Results and discussion}

To verify the validity of proposed algorithm, the traditional travel route recommendation algorithm is compared in this paper. In the traditional travel route recommendation algorithm, the recommendation algorithm which respectively considers POI distance and only considers user interest preference is taken as the measurement standard. Under different time budget B, the traditional algorithm is compared with the proposed algorithm, the travel route recommendation algorithm based on POI distance and user interest preference proposed in this paper, and the experimental results are shown in Figure 3-4.

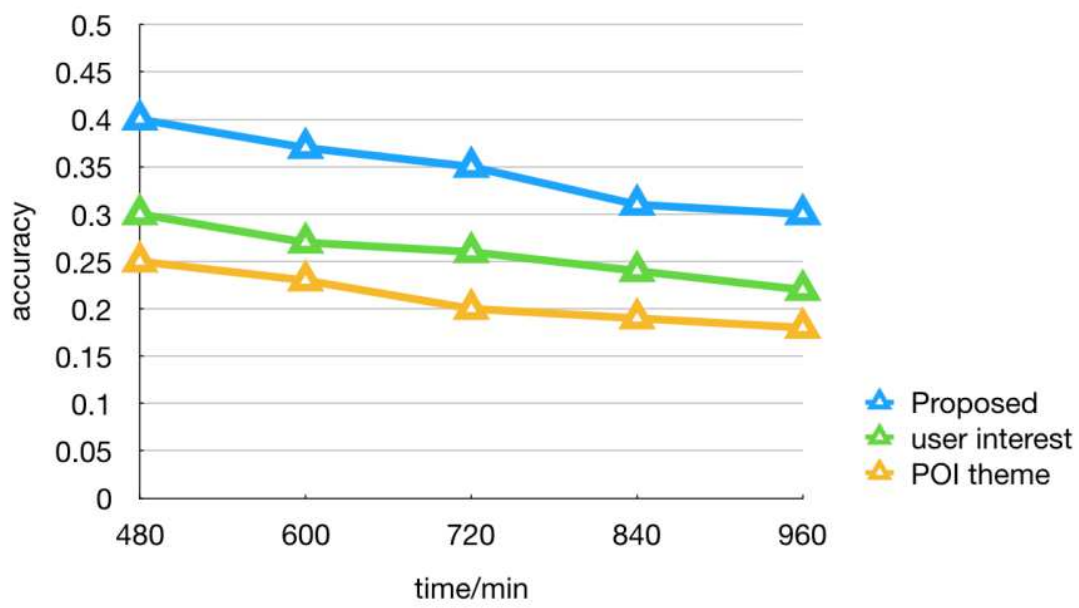

Figure 3 Results of accuracy 


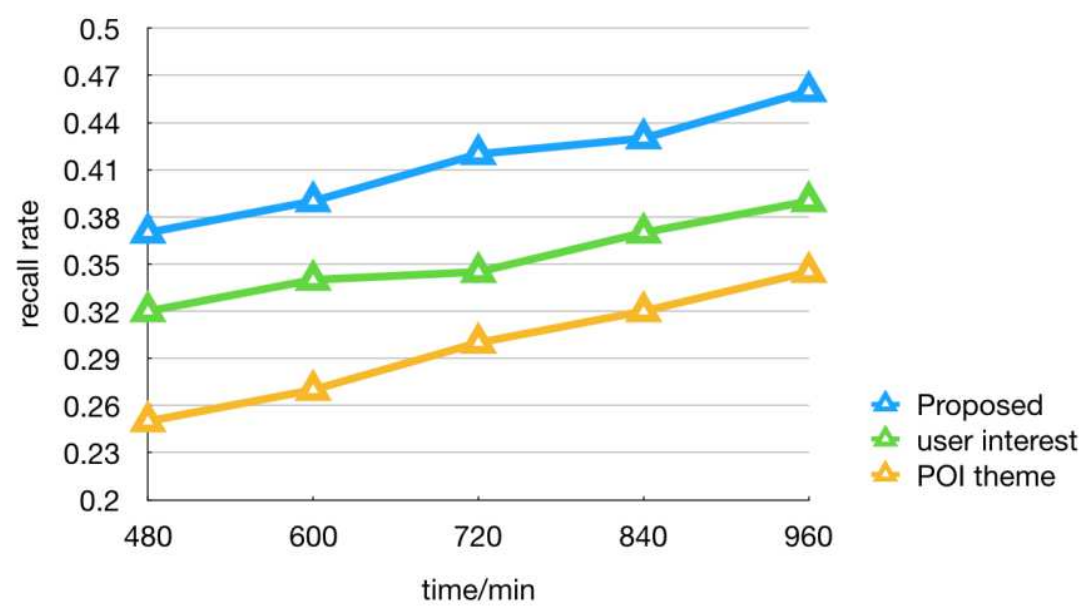

Figure 4 Results of recall rate

In terms of accuracy, it can be seen from Fig. 3 that the proposed algorithm has higher accuracy than the traditional algorithm that only considers the user interest and the POI theme. In terms of recall rate, as shown in Figure 4, the proposed algorithm has a higher recall rate than the traditional algorithm that only considers user interest and the POI theme. One of the influencing factors is that the algorithm proposed and the algorithm that only considers user interest take into account the user's interest, because users prefer to visit places of interest to them. The high accuracy and high recall rate of the proposed algorithm indicate that the proposed algorithm can more accurately recommend the route reflecting the user's real travel sequence.

\section{Conclusion}

In this paper, a tourism recommendation model is established based on the orientation problem, then a personalized travel route recommendation algorithm is proposed. The algorithm takes POI distance and user interest preference into consideration to recommend the most suitable route to users, and implements the travel route recommendation framework by using Flickr photo set with geographic label. Finally, it is verified by experiments that the proposed algorithm has higher recommendation accuracy and recall rate than the traditional algorithm that only considers the POI distance or only considers the user's interest preference. The next work is to further study the intelligent optimization algorithm to solve the directional problem, so as to improve the algorithm operation efficiency and reduce the overhead.

\section{ABBREVIATIONS}

GPS: Global Position System

DBSCAN: Density-Based Spatial Clustering of Applications with Noise

POI: Point of Interest

WAMDM: Web And Mobile Data Management

\section{ETHICS APPROVAL AND CONSENT TO PARTICIPATE}

Not applicable 


\section{CONSENT FOR PUBLICATION}

Not applicable

\section{AVAILABILITY OF DATA AND MATERIAL}

The labeled dataset used to support the findings of this study are available from the corresponding author upon request.

\section{COMPETING INTERESTS}

The authors declare that they have no competing interests.

\section{FUNDING}

Sichuan Province Educational Informatization Application and Development Research Center Project (JYXX18-030).

\section{AUTHORS' CONTRIBUTIONS}

$\mathrm{XI}$ cheng, as the primary contributor, completed the analysis, experiments and paper writing.

\section{ACKNOWLEDGEMENTS}

TangSeng Huang helped perform the analysis with constructive discussions.

\section{References}

1. Alhamid M F, Rawashdeh M, Dong H, et al. Exploring latent preferences for context-aware personalized recommendation systems[J]. IEEE Transactions on Human-Machine Systems, 2016, 46(4): 615-623.

2. Klašnja-Milićević A, Ivanović M, Vesin B, et al. Enhancing e-learning systems with personalized recommendation based on collaborative tagging techniques[J]. Applied Intelligence, 2018, 48(6): 1519-1535.

3. Ayata D, Yaslan Y, Kamasak M E. Emotion based music recommendation system using wearable physiological sensors[J]. IEEE transactions on consumer electronics, 2018, 64(2): 196-203.

4. Colomo-Palacios R, García-Peñalvo F J, Stantchev V, et al. Towards a social and context-aware mobile recommendation system for tourism[J]. Pervasive and Mobile Computing, 2017, 38: 505-515.

5. Benfares C, El Idrissi Y E B, Amine A. Smart city: Recommendation of personalized services in patrimony tourism[C]//2016 4th IEEE International Colloquium on Information Science and Technology (CiSt). IEEE, 2016: $835-840$.

6. Chen J, Zhang H, He X, et al. Attentive Collaborative Filtering: Multimedia Recommendation with Item and Component-Level Attention[C]// International ACM SIGIR Conference on Research and Development in Information Retrieval. ACM, 2017:335-344.

7. Cui L, Dong L, Fu X, et al. A video recommendation algorithm based on the combination of video content and social network[J]. Concurrency and Computation: Practice and Experience, 2017, 29(14): e3900. 
8. HUANG Y, Jinxin Y, Wei S U N. Research of hybrid recommendation algorithm based on improved bipartite network and expert trust[J]. Value Engineering, 2017, 36(19): 160-164.

9. Liu Q, Chen E, Xiong H, et al. A Cocktail Approach for Travel Package Recommendation[J]. IEEE Transactions on Knowledge \& Data Engineering, 2013, 26(2):278-293.

10. Tan C, Liu Q, Chen E, et al. Object-Oriented Travel Package Recommendation[J]. Acm Transactions on Intelligent Systems \& Technology, 2014, 5(3):1-26.

11. Lee C M, Thomas J J. Travel Route Recommendation Based on Geotagged Photo Metadata[C]// International Visual Informatics Conference. Springer, Cham, 2017, 297-308.

12. Nie LQ, Song XM, Chua TS. Learning from multiple social networks. In: Proc. of the Synthesis Lectures on Information Concepts, Retrieval, and Services. Morgan \& Claypool Publishers, 2016.

13. Yin PF, Ye M, Lee WC, Li ZH. Mining GPS data for trajectory recommendation. In: Proc. of the 18th Pacific-Asia Conf. on PAKDD. Springer-Verlag, 2014. 50-61.

14. Eldawy A, Mokbel MF, Al-Harthi S, Alzaidy A, Tarek K, Ghani S. SHAHED: A MapReduce-based system for querying and visualizing spatio-temporal satellite data. In: Proc. of the ICDE. 2015. 1585-1596.

15. Shen Y, Zhao LG, Fan J. Analysis and visualization for hot spot based route recommendation using short-dated taxi GPS traces. Information, 2015,6(2):134-151.

16. He YB, Tan HY, Luo WM, Feng SZ, Fan JP. MR-DBSCAN: A scalable MapReduce-based DBSCAN algorithm for heavily skewed data. Frontiers of Computer Science, 2014, 8(1):83-99.

17. Li YX, Bailey J, Kulik L. Efficient mining of platoon patterns in trajectory databases. Data Knowledge Engineering, 2015,100: 167-187.

18. Fan Q, Zhang DX, Wu HY, Tan KL. A general and parallel platform for mining co-movement patterns over largescale trajectories. PVLDB, 2016,10(4):313-324.

19. Hasuike T, Katagiri H, Tsubaki H, Tsuda H. A route recommendation system for sightseeing with network optimization and conditional probability. In: Proc. of the SMC. 2015. 2672-2677.

20. Gavalas D, Konstantopoulos C, Mastakas K, et al. Review: Mobile recommender systems in tourism[J]. Journal of Network \& Computer Applications, 2014, 39(1):319-333.

21. Xin C, Gao C, Jensen C S. Mining significant semantic locations from GPS data[M]. VLDB Endowment, 2010. 


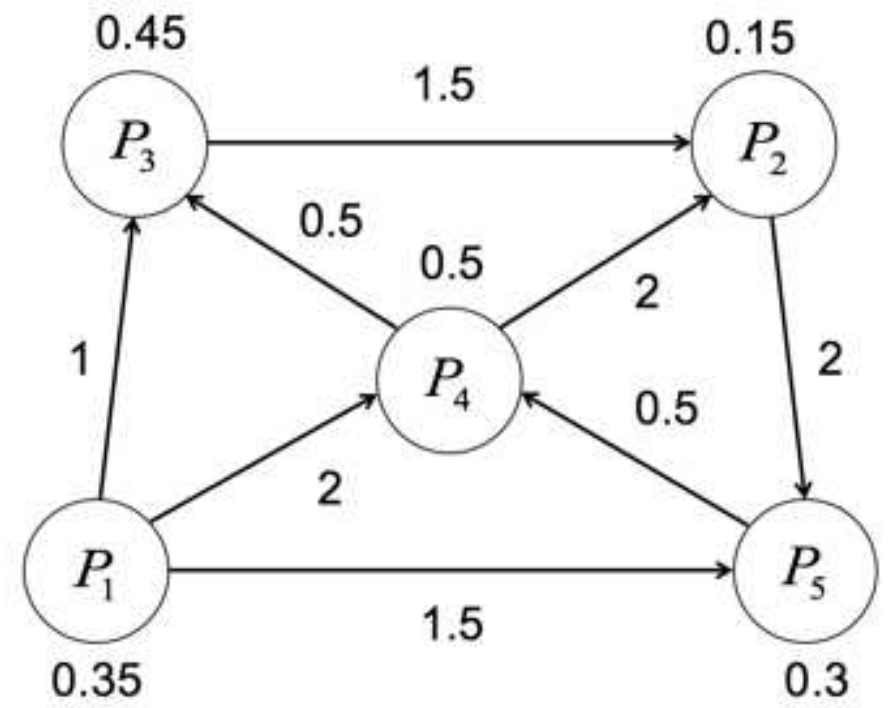

Figure 1

An example of a route recommendation

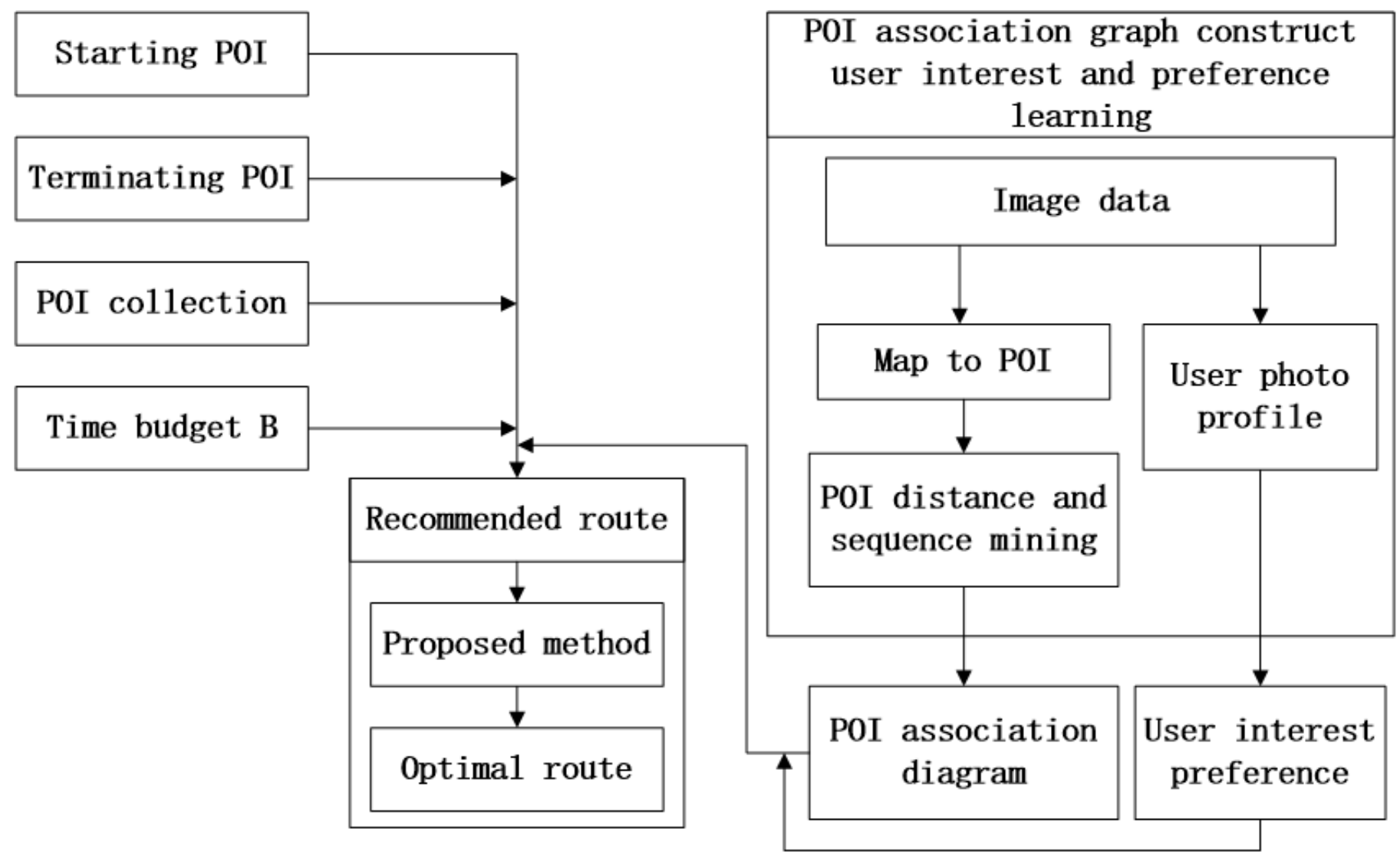


Figure 2

Route recommendation framework

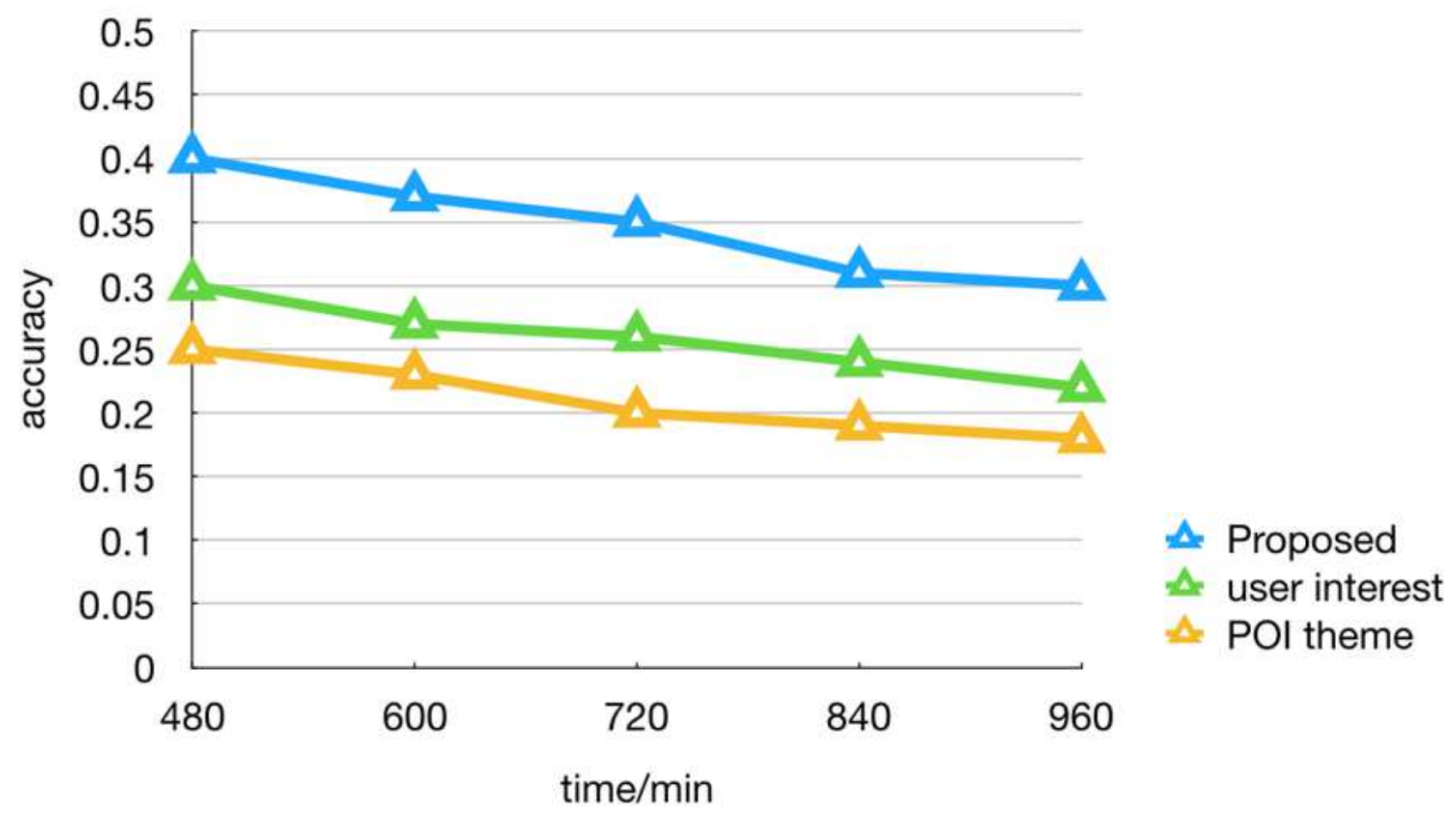

Figure 3

Results of accuracy

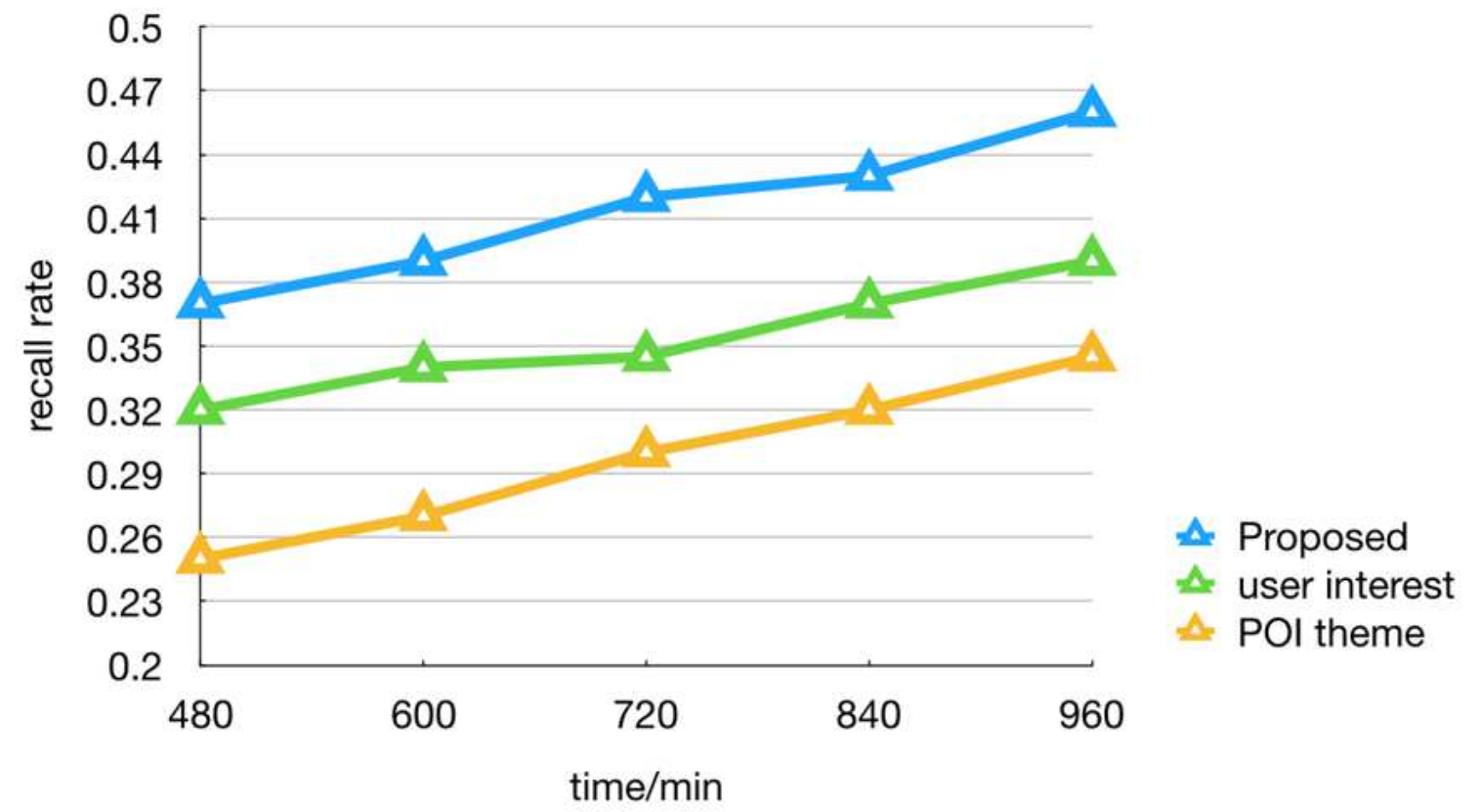


Figure 4

Results of recall rate 